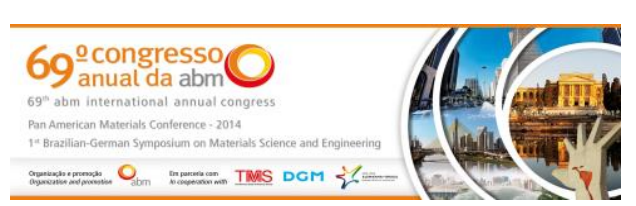

Tema: Materiais com efeito de memória de forma

\title{
EFEITO DO RECOZIMENTO NAS TEMPERATURA DE TRANSFORMAÇÃO DE FASE DE UMA LIGA NiTi*
}

Tadeu Castro da Silva ${ }^{1}$ Daniel Monteiro Rosa ${ }^{2}$ Edson Paulo da Silva²

\section{Resumo}

As ligas $\mathrm{Ni}$-Ti com composição em torno de $55 \%$ de $\mathrm{Ni}$ em peso sofrem influência nas temperaturas de transformação de fase quando submetidos a tratamentos térmicos de recozimento na faixa de temperatura de $300^{\circ} \mathrm{C}$ a $500^{\circ} \mathrm{C}$. O objetivo do presente trabalho é avaliar a influência do tratamento térmico de recozimento a $350^{\circ} \mathrm{C}$ onde os CP's de uma liga NiTi mantidos por tempos de 10 minutos, 1 hora e 24 horas. Os efeitos do tratamento são avaliados com base nas temperaturas de transformação de fase, medida por meio de Calorimetria Diferencial de Varredura (DSC). Os resultados revelaram o maior aumento das temperaturas de transformação de fase para amostra com menor tempo de resfriamento em relação a amostra sem tratamento térmico.

Palavras-chave: Tratamento térmico; Ligas com memória de forma; Calorimetria diferencial de varredura.

\section{EFFECT OF ANNEALING TEMPERATURE ON PHASE TRANSFORMATION OF A LEAGUE NiTi}

\section{Abstract}

The $\mathrm{Ni}-\mathrm{Ti}$ alloys with $55 \%$ w.t. of composition are influenced in the phase change temperatures when they are submitted to the annealing heat treatment in the temperature range of $300 \mathrm{C}$ and $500 \mathrm{C}$. The aim of the current work is to evaluate the influence of the annealing process when the specimen made of NiTi are kept during a period of time between 1 and 24 hours. The effects of the thermal treatment are evaluated taking in account the phase transformation temperatures, measured according to the Differential Scanning Calorimetry (DSC) approach. The results showed the largest increase in temperatures of phase transformation for the sample with lower cooling time than the sample without heat treatment.

Keywords: Thermal treatment; Shape memory alloys; Differential scanning calorimetry.

1 Mestrando em Sistemas Mecatrônicos, Departamento de Engenharia Mecânica, Universidade de Brasília, Brasília, DF, Brasil.

2 Prof. Dr., Departamento de Engenharia Mecânica da Universidade de Brasília, Brasília, DF, Brasil.

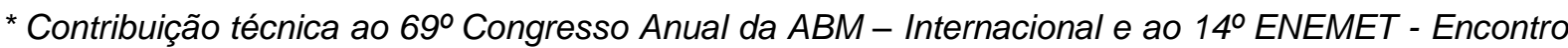
Nacional de Estudantes de Engenharia Metalúrgica, de Materiais e de Minas,21 a 25 de julho de 2014, São Paulo, SP, Brasil.
} 


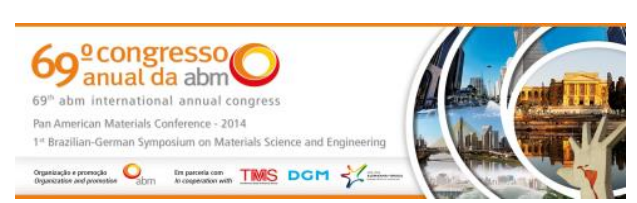

\section{INTRODUÇÃO}

Contrariamente aos materiais convencionais, para os quais variações moderadas de temperatura não afetam suas propriedades mecânicas, as ligas com Memória de Forma (Shape Memory Alloys - SMA), são ligas de materiais que apresentam comportamento termomecânico fortemente dependente da temperatura. Dentre as SMA, as ligas NiTi são as mais intensamente investigadas e aplicadas, devido principalmente às maiores deformações recuperáveis e à sua biocompatibilidade [1-2].

As ligas NiTi possuem quatro temperaturas características, a saber: $\mathrm{Mi}_{\mathrm{i}}$ (temperatura inicial de formação da martensita), $M_{f}$ (temperatura final de formação da martensita); $A_{i}$ (temperatura inicial de formação da austenita) e Af (temperatura final de formação da austenita). Essas temperaturas variam em função da composição química e de tratamentos térmicos apropriados [3-4]. No presente estudo, desenvolveu-se uma análise experimental para avaliação dos efeitos produzidos pelo tratamento térmico de recozimento numa liga $\mathrm{NiTi}$. O resultado mostrou que o maior aumento ocorreu na temperatura final da martensita para amostra $A_{1}$ em relação a Ast, aproximadamente $54 \%$.

\section{MATERIAIS E MÉTODOS}

Para este estudo foram utilizadas quatro amostras da liga Ti55\%Ni em percentual de massa com tamanhos aproximados de $10 \mathrm{~mm}$ de diâmetro por $5 \mathrm{~mm}$ de comprimento. As amostras, designadas como $A_{1}, A_{2}$ e $A_{3}$ foram submetidas a uma temperatura constante de $350^{\circ} \mathrm{C}$, durante 1 hora em um forno experimental. Após este período, o forno foi desligado e as amostras mantidas dentro da câmara de aquecimento por 10 minutos, 1 hora, e 24 horas, respectivamente, para as amostras $A_{1}, A_{2}$ e $A_{3}$. A temperatura de cada amostra foi monitorada por termopares tipo $K$, com isolamento mineral e bainha de aço inoxidável fabricados pela Ecil acoplados a um registrador de dados modelo USB-9162 fabricado pela National Instruments que possibilitou a aquisição das curvas de resfriamento. Após o tratamento térmico, foi realizado o ensaio calorimétrico nas amostras tratadas e em uma amostra sem tratamento (Ast). Neste ensaio foram determinados os valores de temperaturas $A_{i}$, $A_{p}, A_{f}, M_{i}, M_{p}$ e $M_{f}$, bem como o calor latente de transformação de fase através do método das tangentes. Para tanto, foi utilizado um calorímetro diferencial de varredura, modelo DSC-8500 e software Pyris da Perkin Elmer. Este equipamento tem a capacidade de operação em uma faixa de temperatura de $80^{\circ} \mathrm{C}$ a $750^{\circ} \mathrm{C}$ e taxa de aquecimento e resfriamento controlada de 0,01 a $750 \circ \mathrm{C} / \mathrm{min}$ previamente calibrado utilizando padrões dos pontos de fusão do índio e alumínio.

Nas medidas de DSC foi utilizado amostras com massas entre 30 e $32 \mathrm{mg}$ e suporte (porta amostras) de alumínio, sendo aplicado uma taxa de aquecimento e resfriamento controlada de $20^{\circ} \mathrm{C} / \mathrm{min}$ em uma faixa de temperatura de $-20^{\circ} \mathrm{C}$ a $120^{\circ}$ C sob fluxo contínuo de nitrogênio gasoso N2 de alta pureza 99,999\% e vazão de $20 \mathrm{~mL} / \mathrm{min}$.

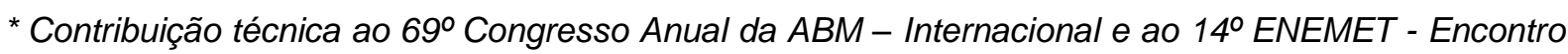
Nacional de Estudantes de Engenharia Metalúrgica, de Materiais e de Minas,21 a 25 de julho de 2014, São Paulo, SP, Brasil.
} 


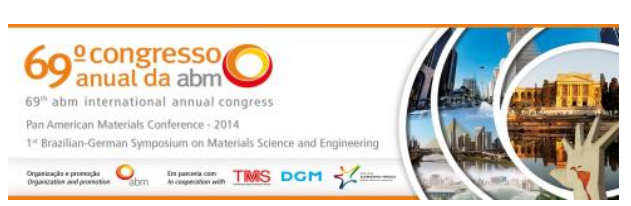

\section{RESULTADOS E DISCUSSÃO}

A Figura 1 mostra o resultado do ensaio realizado no DSC para a amostra Ast, sem tratamento térmico. As duas curvas correspondem ao aquecimento (curva superior) e ao resfriamento (curva inferior), onde observam-se os inícios e fins das transformações: $A_{i}, A_{f}, M_{i}, M_{f}$. além do pico endotérmico $A_{p} \sim 70,19$ e pico exotérmico $\mathrm{Mp} \sim 29,18^{\circ} \mathrm{C}$ aproximadamente.

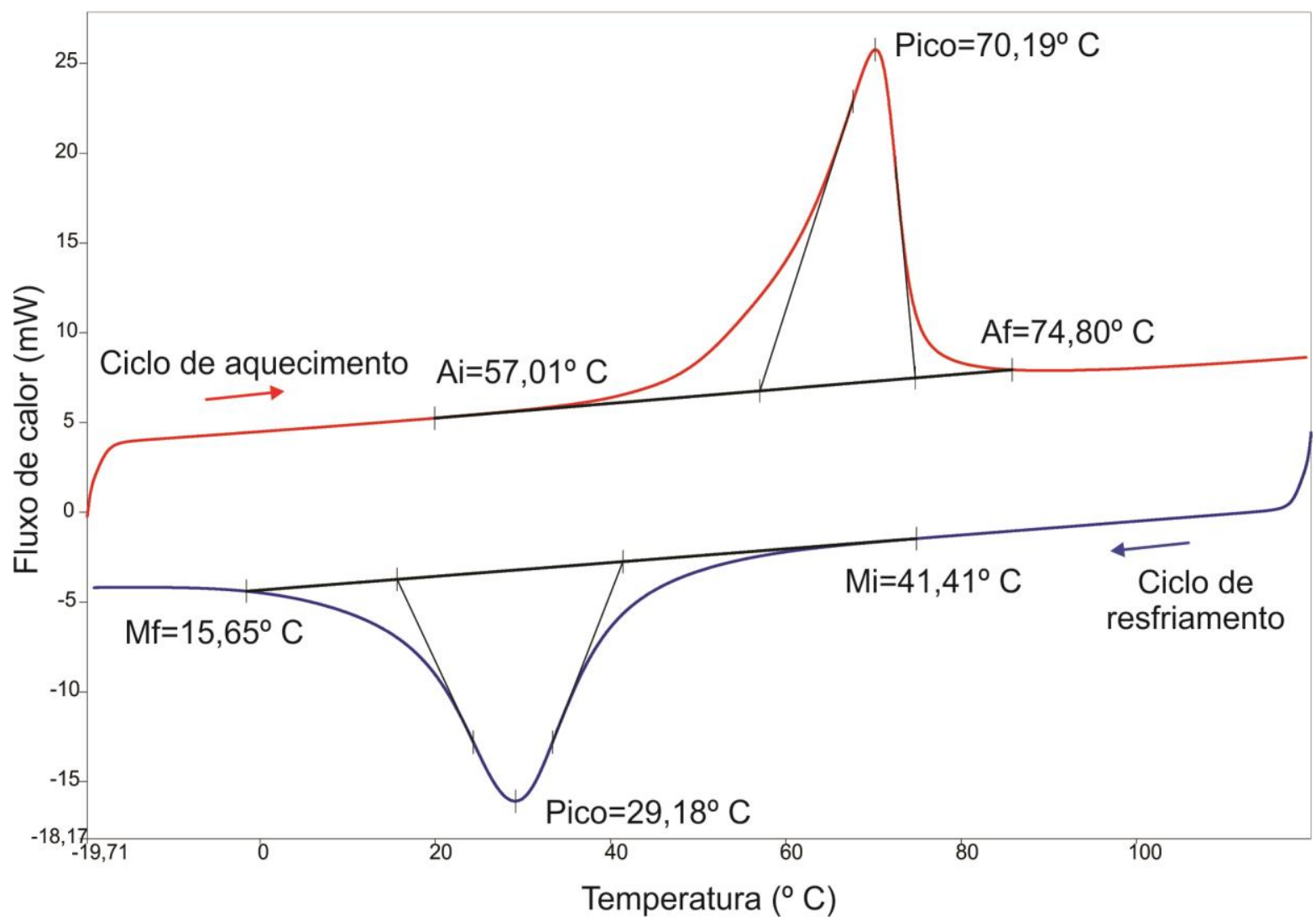

Figura 1. Curvas DSC para a amostra como recebido.

A Tabela 1 mostra as temperaturas $A_{i}, A_{f}, M_{i}, M_{f}$ os picos $A_{p}$ e $M_{p}$, obtidos para as amostras tratadas e para a não tratada.

Tabela 1. Dados obtidos na análise calorimétrica.

\begin{tabular}{c|c|c|c|c|c|c}
\hline Amostras & $\mathrm{A}_{\mathrm{i}}\left({ }^{\circ} \mathrm{C}\right)$ & $\mathrm{A}_{\mathrm{p}}\left({ }^{\circ} \mathrm{C}\right)$ & $\mathrm{A}_{\mathrm{f}}\left({ }^{\circ} \mathrm{C}\right)$ & $\mathrm{M}_{\mathrm{i}}\left({ }^{\circ} \mathrm{C}\right)$ & $\mathrm{M}_{\mathrm{p}}\left({ }^{\circ} \mathrm{C}\right)$ & $\mathrm{M}_{\mathrm{f}}\left({ }^{\circ} \mathrm{C}\right)$ \\
\hline $\mathrm{A}_{\mathrm{ST}}$ & 57,01 & 70,19 & 74,80 & 41,41 & 29,18 & 15,65 \\
$\mathrm{~A}_{1}$ & 59,06 & 71,42 & 77,71 & 44,29 & 37,68 & 24,21 \\
$\mathrm{~A}_{2}$ & 64,53 & 75,64 & 78,81 & 44,21 & 33,01 & 23,32 \\
$\mathrm{~A}_{3}$ & 61,12 & 73,40 & 77,92 & 42,71 & 35,50 & 23,74 \\
\hline
\end{tabular}

As Figuras 2 e 3 mostram os gráficos comparativos de $A_{i}, A_{f}$ e pico endotérmico para as amostras analisadas. Pode ser observado que há uma tendência de deslocamento positivo tanto $A_{i}$ quanto de $A_{f}$ em função do tempo de tratamento da amostra em relação à amostra não tratada (ST).

As Ligas de $\mathrm{Ni}-\mathrm{Ti}$, rica em $\mathrm{Ni}$, melhoram as propriedades de superelasticidade, em resposta a tratamentos térmicos de recozimento de $350^{\circ} \mathrm{C}$ e elevam a força motriz

\footnotetext{
* Contribuição técnica ao 69ำ Congresso Anual da ABM - Internacional e ao 14ํㅡㄹ ENEMET - Encontro Nacional de Estudantes de Engenharia Metalúrgica, de Materiais e de Minas,21 a 25 de julho de 2014, São Paulo, SP, Brasil.
} 
para a precipitação de Ti3Ni4 [5]. Esses precipitados na matriz afetam diretamente a faixa de histerese e o amortecimento da liga Ni-Ti.

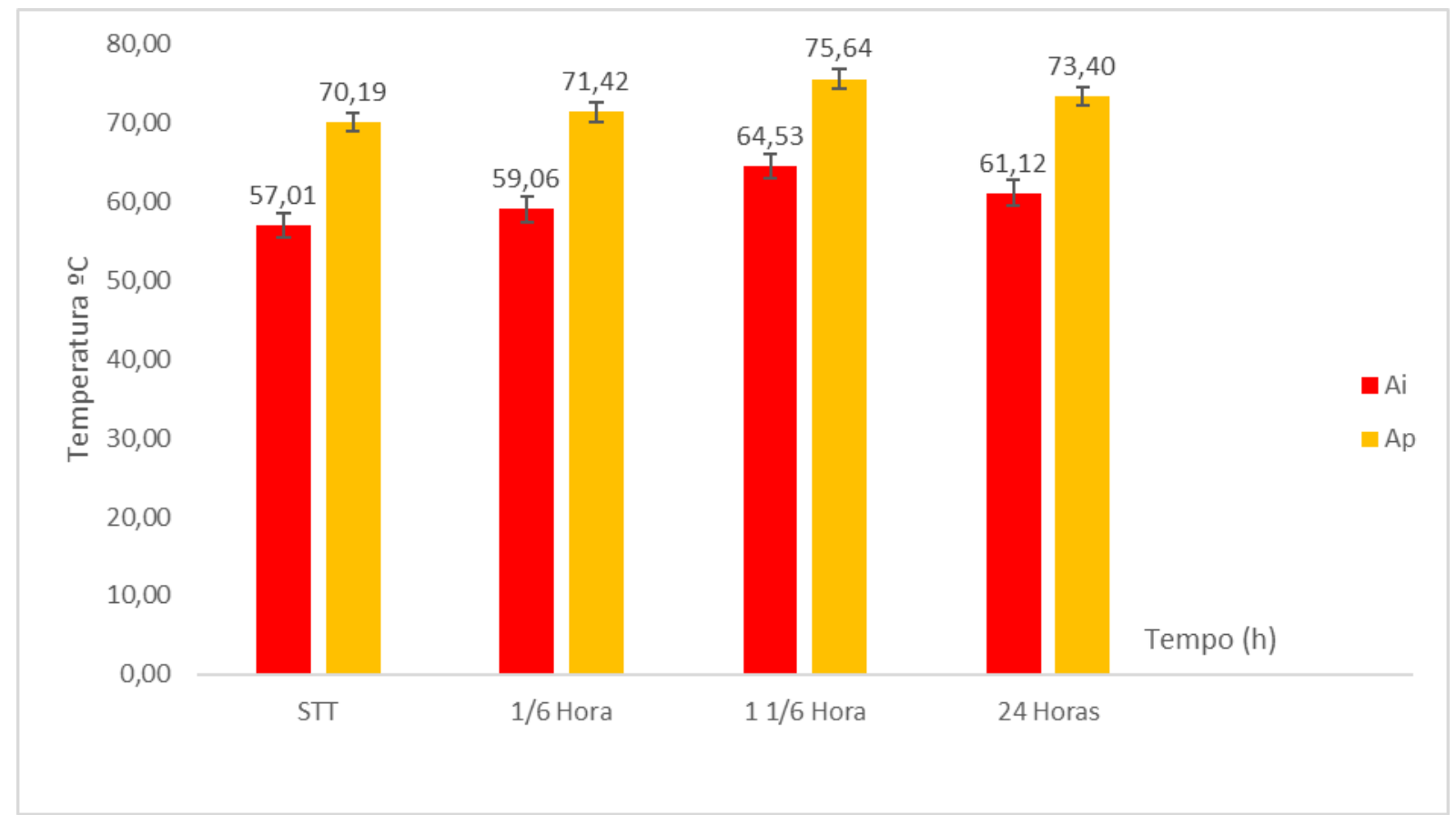

Figura 2. $A_{i}$ e $A_{p}$ para as quatro amostras analisadas.

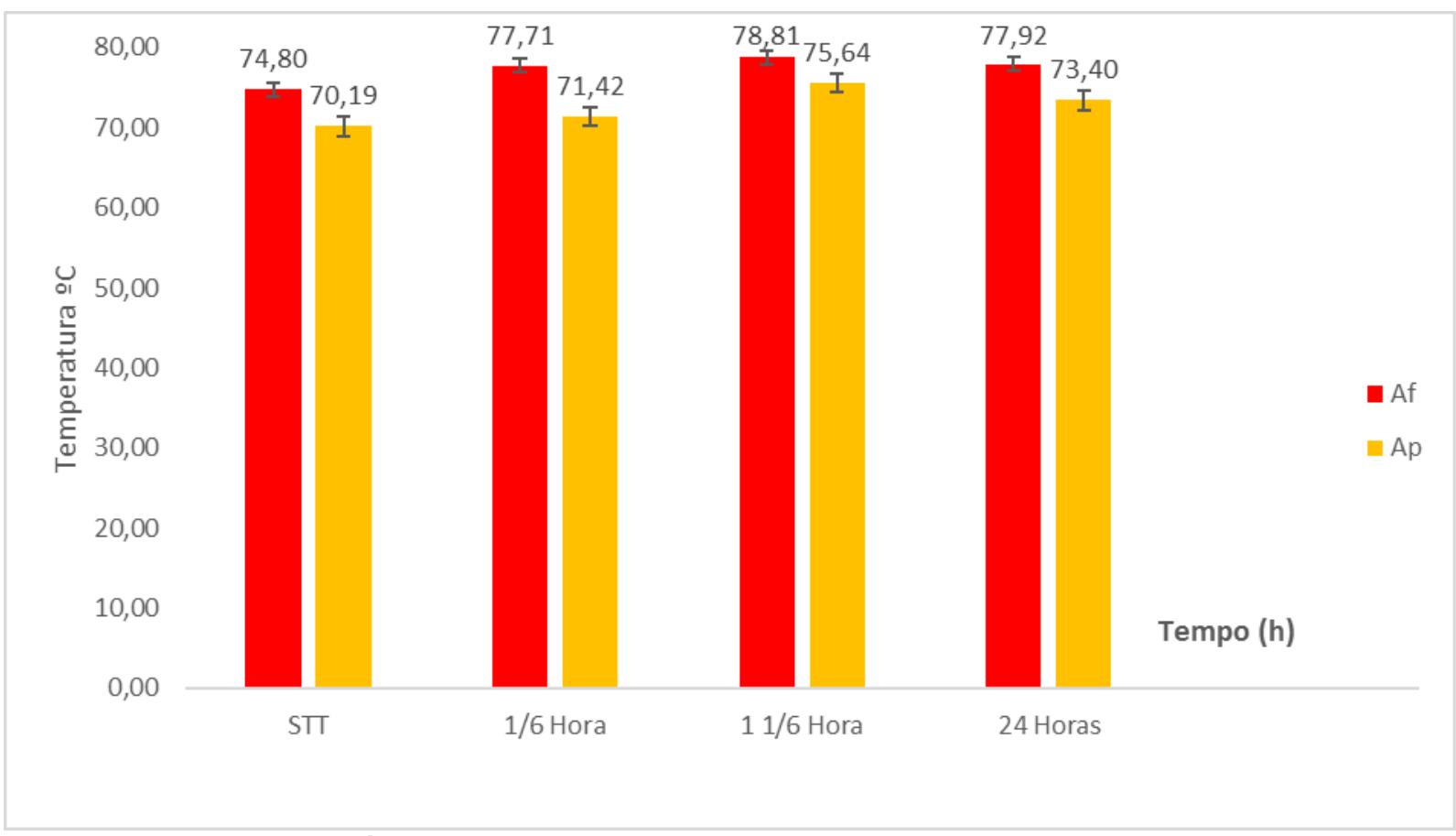

Figura 3. $A_{f}$ e $A_{p}$ para as quatro amostras analisadas.

As Figuras 4 e 5 mostram os gráficos comparativos de $\mathrm{M}_{\mathrm{i}} \mathrm{M}_{\mathrm{f}}$ e pico exotérmico para as amostras analisadas. Pode ser observado que há uma tendência de deslocamento positivo de $\mathrm{Mi}_{\mathrm{i}}$ com uma diminuição para a amostra tratada por $24 \mathrm{~h}$. Esta mesma tendência pode ser observada para o final da transformação da martensita mesmo para a amostra tratada por $24 \mathrm{~h}$.

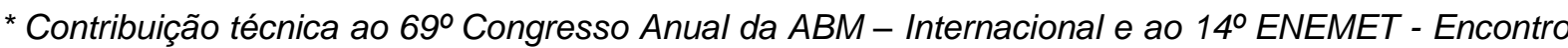
Nacional de Estudantes de Engenharia Metalúrgica, de Materiais e de Minas,21 a 25 de julho de 2014, São Paulo, SP, Brasil.
} 
O aumento das temperaturas de transformação de fase pode ocorre em razão do alívio de tensões provenientes da redução de discordâncias permitindo assim, a nucleação da martensita em temperaturas mais altas [6]. Outro aspecto importante que deve ser considerado é o efeito da formação de precipitados de $\mathrm{Ti}_{3} \mathrm{Ni}_{4}$ em razão do tratamento térmico [7].

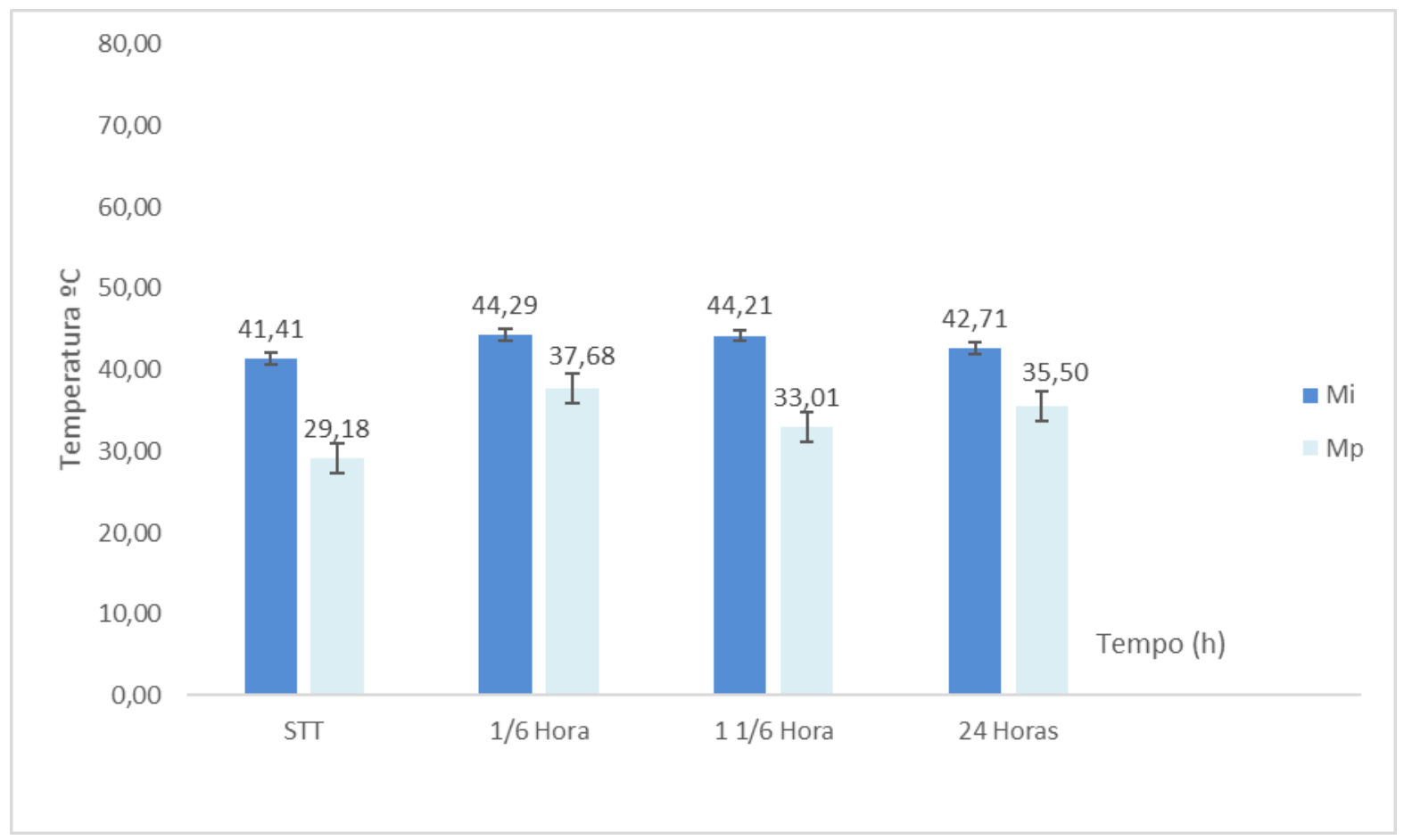

Figura 4. $M_{i}$ e $M_{p}$ para as quatro amostras analisadas.

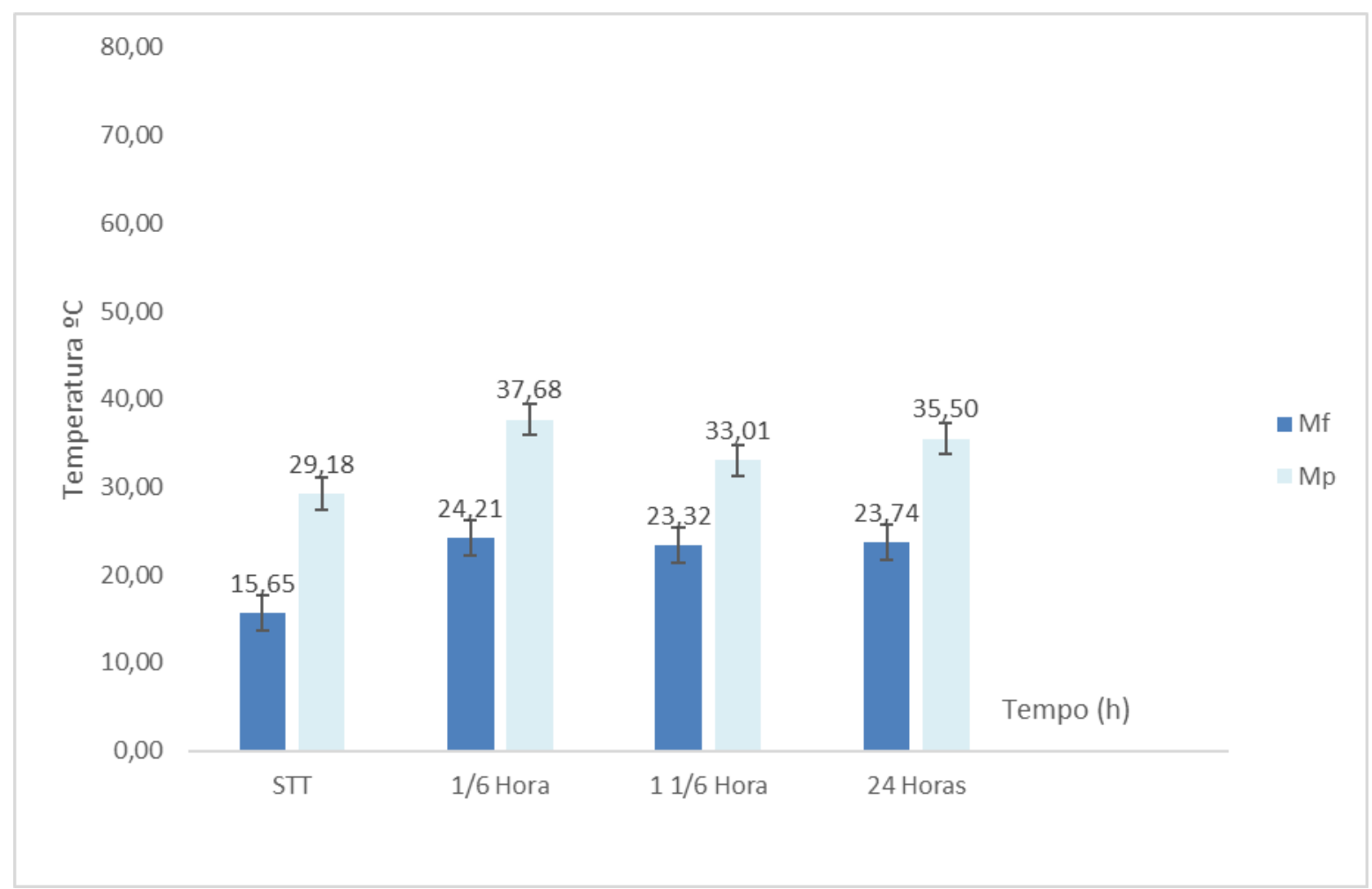

Figura 5. $M_{f}$ e $M_{p}$ para as quatro amostras analisadas.

* Contribuição técnica ao $69^{\circ}$ Congresso Anual da ABM - Internacional e ao 14ํㅡㄹ ENEMET - Encontro Nacional de Estudantes de Engenharia Metalúrgica, de Materiais e de Minas,21 a 25 de julho de 2014, São Paulo, SP, Brasil. 


\section{CONCLUSÃO}

Nesse estudo, o tratamento térmico de recozimento alterou significativamente as temperaturas de transformação de fase presentes na liga. Verificou-se que as propriedades da liga são influenciadas pelo tempo de tratamento térmico e que o maior aumento ocorre no final da martensita para o menor tempo de resfriamento.

\section{Agradecimentos}

O autor ${ }^{1}$ agradece à CAPES/MEC pelo auxílio financeiro para realização da pesquisa.

\section{REFERÊNCIAS}

1 Funakubo H.1987, Shape Memory Alloys, Ed. Hiroyasu Funakubo,Tokyo: University of Tokyo, 1987.

2 Funakubo, H., Gordon \& Bleach, Shape Memory Alloys. New York, 1987.

3 Miyazaky S., Sandström R., Wei, Z.G. Review Shape-memory materials and hybrid composites for smart systems. Journal of materials science 33. 1998.

4 Otsuka K, Wayman CM. Shape memory materials, Cambridge University Press. Cambridge.1998.

5 Otsuka, K.Engineering aspects of shape memory alloys edited by T W Dueriget al (Butterworth-Heinemann Ltd., England, 1990).

6 Santos CML. Ligas Ni-Ti ricas em Ni Tratamento térmico, Termomecânico e Efeito de micromemória de forma,156p. [Tese de Doutorado], Rio de Janeiro. 2006.

7 Villamarin EB. Estudo das variações microestruturais de uma liga pseudoelástica de $\mathrm{Ni}$ Ti tratada termicamente [Dissertação de mestrado]. Brasília, Universidade de Brasília, 2013.

\footnotetext{
* Contribuição técnica ao $69^{\circ}$ Congresso Anual da ABM - Internacional e ao 14ํㅡㄹ ENEMET - Encontro Nacional de Estudantes de Engenharia Metalúrgica, de Materiais e de Minas,21 a 25 de julho de 2014, São Paulo, SP, Brasil.
} 